Archive for

Organic Chemistry

Arkivoc 2020, part vi, 168-180

\title{
C-Alkyl-bis-phosphoryl chelating systems for the potential recovery of strategic metals
}

\author{
Abdoul Razak Halidou Dougourikoye, ${ }^{a, b}$ Rachida Babouri, ${ }^{a}$ Jean-Noël Volle, ${ }^{\text {a }}$ Amadou TidJani Ilagouma, \\ Jean-Luc Pirat, ${ }^{a}$ and David Virieux ${ }^{a^{*}}$ \\ a ICGM, Univ. Montpellier, ENSCM, CNRS, Montpellier, France \\ ${ }^{b}$ Département de Chimie, Faculté des Sciences, Université Abdou Moumouni, B.P. 10662, Niamey, Niger \\ E-mail: david.virieux@enscm.fr
}

Received 03-26-2020

Accepted 04-27-2020

Published on line $05-10-2020$

\section{Abstract}

Methylene-bis-phosphonic acid and derivatives are used as complexing agents, diagnostics, therapeutics and show interesting virustatic properties. We describe the syntheses of mono- and di-substituted bisphosphonates and mono- and disubstituted diethyl ((diphenylphosphoryl)methyl)phosphonate as possible chelating systems for the recovery of strategic metals.

$$
\begin{aligned}
& \mathrm{R}^{1}, \mathrm{R}^{2}=\text { OEt } \\
& \mathrm{R}^{1}=\text { OEt; } \mathrm{R}^{2}=\mathrm{Ph}
\end{aligned}
$$<smiles>[R12]P=[R2]P=O</smiles>

Ö

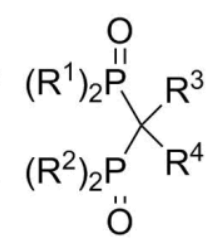

Michaelis-Arbuzov and Michaelis-Becker type reactions

Keywords: Methane-bisphosphonic acid and derivatives, diethyl((diphenylphosphoryl) methyl)phosphonate, chelating systems 


\section{Introduction}

Bisphosphonates (BPs) were introduced first in 1865 by Nikolay Menshutkin, a Russian scientist from Saint Petersburg State University. ${ }^{1}$ German chemists then described BPs as effective anti-scaling agents. ${ }^{2}$ Today, substituted bisphosphonates are mostly applied for medical purposes when calcium metabolism is affected (treatment of Paget's disease, osteoporosis, metastatic bone disease, multiple myeloma and several rare bone diseases, and as antibiotics, hormones and anti-cancer drugs). ${ }^{3,4,5,6,7,8}$

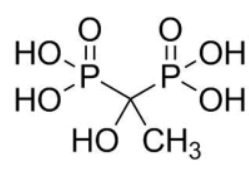

Etidronate Early BPs

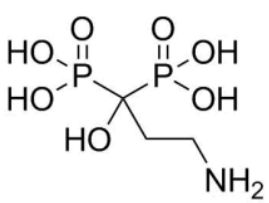

Pamidronate Second generation

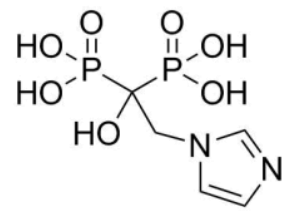

Zoledronate Third generation

Figure 1. BPs that have been approved for clinical use.

Currently, other areas of research are investigating the applicability of BPs for wastewater treatment or valuable metals extraction. For instance, the demand for Rare Earth Elements (REEs), a group of 17 metals, including 15 lanthanides, yttrium and scandium, have become of strategic importance for hi-tech applications (electronic devices, electric cars) ${ }^{9}$ and are considered as critical materials on a worldwide market scale for future technologies. ${ }^{10}$

The P-C-P backbone of BPs and the alkyl side chains on the central carbon atom have a determinant role in their physicochemical properties, each leading to different solubilities in solvents or their affinity to metal cations. All of this makes BPs very promising compounds for a large number of applications.

\section{Results and Discussion}

As part of our ongoing efforts in the synthesis of original and active phosphorus compounds, we, herein, report the preparation of various BPs and mixed mono- and di-substituted phosphine oxide-phosphonates for potential applications in the recovery of strategic metals. Even if REEs are important elements, such syntheses have to be cost effective for industrial purposes. Therefore, this work was focused on the use of the simplest and least expensive methods to obtain BP-complexing systems which would allow the recycling of rare earth metals. We first synthesized tetraethyl methylene bisphosphonate, and then carried out substitution reactions on the methylene bridge with functional groups in order to provide the desired complementary properties.

\section{Syntheses of tetraethyl methylene bis(phosphonate)}

Two different approaches have been taken to obtain the desired tetraethyl methylene bis(phosphonate) under the best conditions.

In the first method, dibromomethane and an excess of triethyl phosphite were mixed and heated to $150^{\circ} \mathrm{C} .{ }^{11}$ The reaction was stopped after 48 hours following total consumption of the triethyl phosphite. At the end of the reaction, the ${ }^{31} \mathrm{P}$ NMR spectrum indicated that fairly large amounts of by-products were formed, 
most of which was a byproduct of the Arbuzov reaction, diethyl ethylphosphonate (69\%). Distillation with a Kugelrohr apparatus allowed us to obtain the desired compound (1) in 27\% yield (Scheme 1).

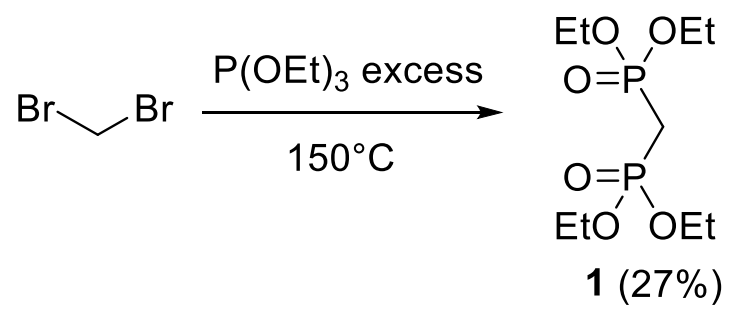

Scheme 1: Tetraethyl methylene bis(phosphonate) synthesis by direct double Arbuzov reaction.

In the second approach, tetraethyl methylenebis(phosphonate) was obtained in two steps. The first step consisted of the reaction of metallic sodium with an excess of diethyl phosphite, followed by the addition of dichloromethane in the second step (Scheme 2). ${ }^{12,13}$
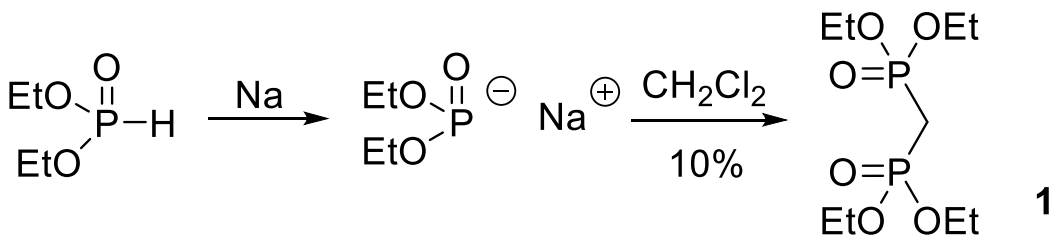

Scheme 2. Tetraethyl methylene bis(phosphonate), synthesis by double Michaelis-Becker reaction on dichloromethane.

Product (1) was obtained after distillation using a molecular pump at $10^{-4} \mathrm{mbar}$, in $10 \%$ yield. The first method was preferably used to synthesize tetraethyl methylene bis(phosphonate) (1).

\section{Substitution reactions on methylene bridge of BPs}

Generally, the alkylation reactions of the methylene bridge of BPs have been carried out using sodium, ${ }^{[7]}$ sodium hydride with ${ }^{14}$ or without ${ }^{15}$ crown ethers, and with cesium carbonate or potassium carbonate under microwave activation. ${ }^{17}$ The mono-alkyl predominating BP was generally obtained in the presence of the dialkyl species.

We decided to carry out alkylation reactions with different alkyl halides, including those having aromatic moieties or long alkyl chains to avoid cyclization reactions when $\alpha, \omega$-alkyl dihalides were used (Scheme 3 ). Sodium hydride was used as the base and dry THF as the solvent. After reactions at room temperature or $50^{\circ} \mathrm{C}$ (see Table 1), the expected compounds were obtained by purification using silica gel chromatography. The substituted bisphosphonates were isolated in moderate to quantitative yields. It can be noticed that selectivity (mono vs double substitution) was hardly controlled when using small alkyl halides (Table 1, entries 1 and 2), and the reaction was pushed to the formation of the disubstituted derivatives (3a-b) in nearly quantitative yields. 


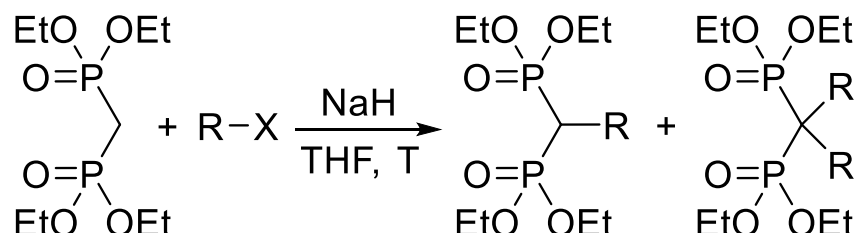

1

2

3

Scheme 3. Alkylation reaction of the methylene bridge of the BP (1).

Table 1. Alkylation reaction of the methylene bridge of the BP (1)

\begin{tabular}{cccccc}
\hline Entry & $\mathrm{R}-\mathrm{X}$ & $\mathrm{T}^{\circ} \mathrm{C}$ & Product & Yield 2 & Yield 3 \\
\hline 1 & $\mathrm{CH}_{3} \mathrm{l}$ (excess, 4.4 eq) & 20 & a & $0 \%$ & $99 \%$ \\
2 & $\mathrm{EtBr}$ (excess, 4.4 eq) & 20 & $\mathbf{b}$ & $0 \%$ & $98 \%$ \\
3 & $\mathrm{Br}-\left(\mathrm{CH}_{2}\right)_{8}-\mathrm{Br}$ & 20 & $\mathbf{c}$ & $21 \%$ & - \\
4 & $\mathrm{CH}_{3}-\left(\mathrm{CH}_{2}\right)_{7}-\mathrm{Br}$ & 50 & $\mathbf{d}$ & $28 \%$ & - \\
5 & $p-\mathrm{CH}_{3}-\mathrm{C}_{6} \mathrm{H}_{4}-\mathrm{CH}_{2}-\mathrm{Br}$ & 50 & $\mathbf{e}$ & $58 \%$ & $3 \%$ \\
6 & $\mathrm{Br}-\mathrm{CH}_{2}-\left(\mathrm{C}_{6} \mathrm{H}_{4}\right)_{8}-\mathrm{CH}_{2}-\mathrm{Br}$ & 20 & $\mathbf{f}$ & $38 \%$ & $5 \%$ \\
\hline
\end{tabular}

To avoid the formation of symmetrical disubstituted BPs (3), we then carried out the synthesis of vinylene bisphosphonate (5) whose reduction gave the methylated BPs (2a) (Scheme 4). ${ }^{18}$

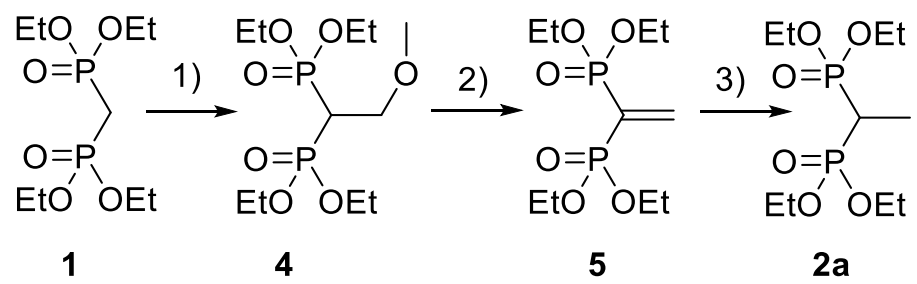

1) $\left(\mathrm{CH}_{2} \mathrm{O}\right) \mathrm{n}, \mathrm{Et}_{2} \mathrm{NH} / \mathrm{MeOH}$, reflux, $24 \mathrm{~h}, 94 \%$, 2) PTSA, Toluene, reflux, $\left.24 \mathrm{~h}, 47 \%, 3\right) \mathrm{H}_{2} 1 \mathrm{~atm}, \mathrm{Pd} / \mathrm{C}, \mathrm{rt}, 96 \%$.

Scheme 4. Selective synthesis of tetraethyl ethane-1,1-diylbis(phosphonate) (2a).

Alkylation of 2a was performed by reaction with sodium hydride, followed by a nucleophilic substitution on $p$-methylbenzyl bromide (Scheme 5). The resulting BP (6) was isolated in $69 \%$ yield, in spite of the hindered character of the central carbon atom.

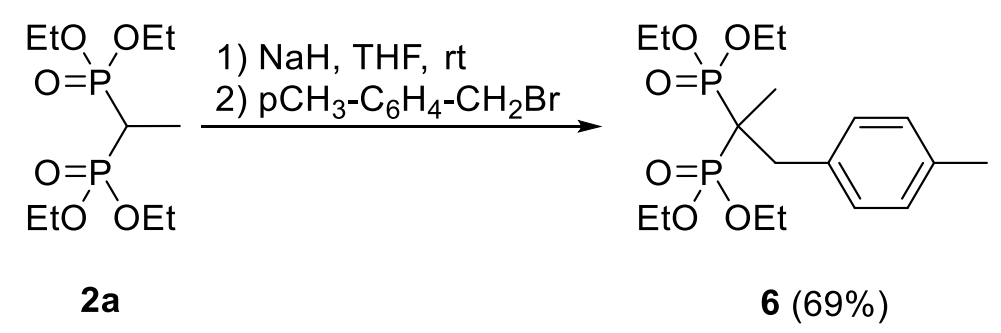

Scheme 5. Synthesis of the dialkylated compounds. 
We then tried to introduce an octyl chain from BP (2a) (Scheme 6). Unfortunately, we did not succeed in forming the expected product (8). On the other hand, we isolated ethyl-substituted BP (7) in low yield. The latter resulted from a nucleophilic attack of the phosphoryl carbanion on the ethyl group of another molecule of $\mathbf{2 a}$, the phosphonate group playing the role of leaving group.

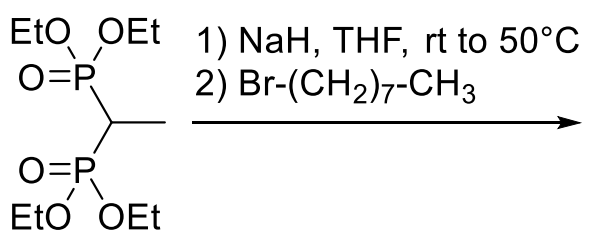

2a

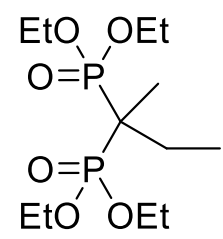

$7(3 \%)$

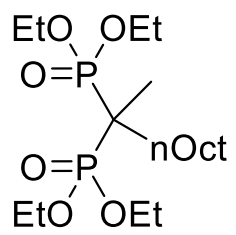

8 (not isolated)

Scheme 6. Alkylation of the monomethyl BP (2a).

Subsequently, we synthesized dialkyl-BP (8) using the reverse sequence (Scheme 7). Alkylation of $\mathbf{2 d}$ by methyl iodide led to BP (8) which was isolated in 75\% yield. Therefore, it appeared noticeably more effective to introduce the largest substituent first, to control the mono-substitution vs the di-substitution, followed by the introduction of the smallest substituent once the reactive system was sterically hindered.

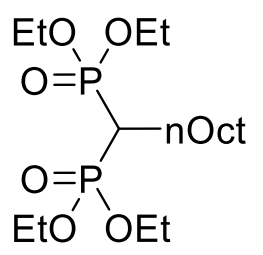

2d

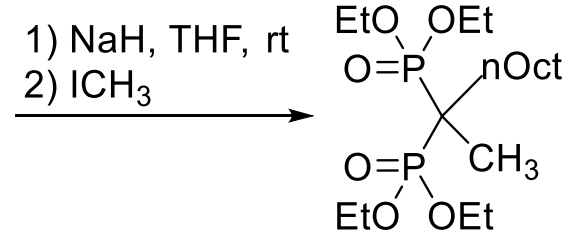

$8(75 \%)$

Scheme 7. Scheme: Synthesis of disubstituted BP (8).

\section{Synthesis of diethyl ((diphenylphosphoryl)methyl)phosphonate}

Mixed phosphine oxide-phosphonates were obtained through a 5-step sequence from chlorodiphenylphosphine (9) (Scheme 8). The first step was the hydrolysis of 9 to give the secondary phosphine oxide (10) which was isolated in $88 \%$ yield. Functionalization of $\mathbf{1 0}$ was accomplished by the reaction of an excess of paraformaldehyde in the presence of catalytic amounts of triethylamine at $80{ }^{\circ} \mathrm{C}$. After 7 hours of reaction, the ${ }^{31} \mathrm{P}$ NMR showed the disappearance of the starting reagent and the formation of a broad signal for diphenyl hydroxymethylphosphine oxide (11). Consecutive treatment of the crude by hot ethanol cleaved the polymeric acetals of 11, usually formed by consecutive reaction with the excess of formaldehyde. Phosphine oxide (11) was then isolated in $94 \%$ yield and it could be used without additional purification.

Transformation of the alcohol function of $\mathbf{1 1}$ into a good leaving group was accomplished by the reaction with para-toluene sulfonyl chloride $(\mathrm{TsCl})$ in the presence of triethylamine. After treatment, the tosylate (12) was obtained quantitatively. The bromine derivative (13) was obtained by Finkelstein reaction using sodium bromide in acetone. The mixture was heated to $75^{\circ} \mathrm{C}$ while stirring for one week. A larger-scale reaction ( 12.8 $\mathrm{g}$ of 12) required 2 weeks to reach completion. After recrystallization, the bromide derivative (13) was obtained in quantitative yield. An Arbuzov reaction with triethyl phosphite permitted the formation of diethyl diphenylphosphinoxymethyl phosphonate (14). 
Previous results in our lab have shown that the reaction is much more efficient with the bromine derivative than with the chlorine one. The ${ }^{31} \mathrm{P}$ NMR spectra showed the presence of two doublets corresponding to the two different phosphorus atoms. Each phosphorus showed a doublet with a coupling constant of $14.4 \mathrm{~Hz}$.

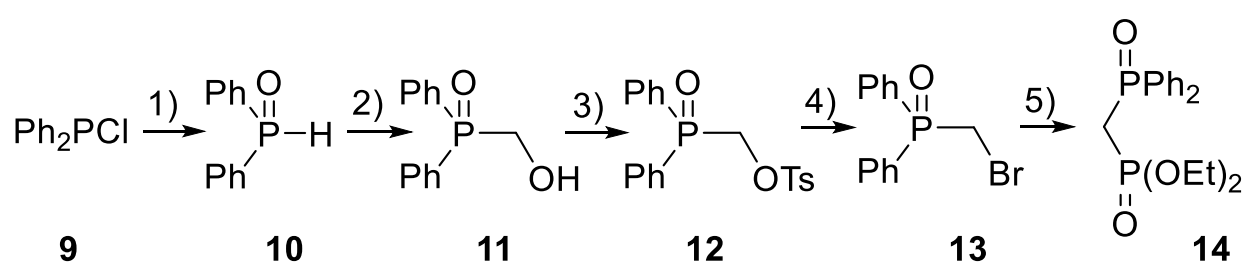

1) $\mathrm{H}_{2} \mathrm{O}, \mathrm{THF}, 0^{\circ} \mathrm{C}$ to $\mathrm{RT}, 88 \%$; 2) $\left.\mathrm{H}\left(\mathrm{CH}_{2} \mathrm{O}\right) \mathrm{nH}, \mathrm{Et}_{3} \mathrm{~N}, 80^{\circ} \mathrm{C}, 94 \% ; 3\right) \mathrm{TsCl}^{\mathrm{E}} \mathrm{Et}_{3} \mathrm{~N}, \mathrm{DCM}, 100 \%$; 4$) \mathrm{NaBr}, \mathrm{CH}_{3} \mathrm{CN}$, $100 \%$; 5) $\mathrm{P}(\mathrm{OEt})_{3}$ excess, reflux, $69 \%$.

Scheme 8. Synthesis of diethyl ((diphenylphosphoryl)methyl) phosphonate (14).

\section{Alkylation of the methylene bridge of the diethyl ((diphenylphosphoryl)methyl)phosphonate (BP derivatives)}

As already illustrated with BPs (2) and (3), syntheses of substituted phosphine oxide-phosphonates (15) were carried out using the same conditions (Scheme 9). Reactions of $\mathbf{1 4}$ with sodium hydride for the deprotonation of the methylene bridge, and then substitution reactions with various alkyl halides (para-bromomethyltoluene and 1,4-bis(bromomethyl)benzene were attempted. Monosubstituted 15a was isolated in $32 \%$ yield and $\mathbf{1 5 b}$ in $30 \%$ yield after purification by chromatography on silica gel (Table 2 ).

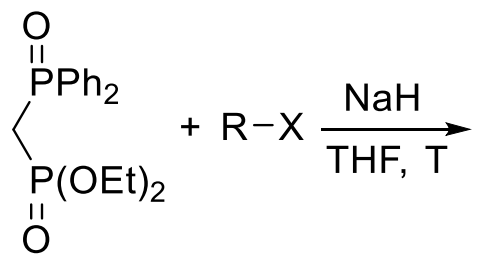

14<smiles>[R6]O[P+](=O)C([R])[Po](=O)[O-]</smiles>

$15 a-b$

Scheme 9. Alkylation reaction of the methylene bridge of the BP derivatives.

Table 2. Alkylation reaction of the methylene bridge of the BP derivatives.

\begin{tabular}{ccccc}
\hline Entry & $\mathrm{R}-\mathrm{X}$ & $\mathrm{T}{ }^{\circ} \mathrm{C}$ & Product & Yield (\%) \\
\hline 1 & $p-\mathrm{CH}_{3}-\mathrm{C}_{6} \mathrm{H}_{4}-\mathrm{CH}_{2}-\mathrm{Br}$ & $50{ }^{\circ} \mathrm{C}$ & $\mathbf{1 5 a}$ & $32 \%$ \\
2 & $\mathrm{Br}-\mathrm{CH}_{2}-\left(\mathrm{C}_{6} \mathrm{H}_{4}\right)_{8}-\mathrm{CH}_{2}-\mathrm{Br}$ & $20{ }^{\circ} \mathrm{C}$ & $\mathbf{1 5 b}$ & $30 \%$ \\
\hline
\end{tabular}

\section{Conclusions}

We were able to synthesize new bisphosphonates and mixed phosphonate-phosphine oxides using MichaelisArbuzov and Michaelis-Becker reactions. Such compounds were easily mono- and di-substituted on the 
methylene bridge, thanks to alkylation reactions with various alkyl halides or by selective introduction of a methyl group from vinylene bisphosphonate. Further developments will be reported in due course and will focus on the recovery of strategic metals.

\section{Experimental Section}

General. ${ }^{1} \mathrm{H}-\mathrm{NMR}$ spectra were recorded on a BRUKER Ultrashield 400 plus $(400.13 \mathrm{MHz})$ instrument. The chemical shifts are expressed in parts per million $(\mathrm{ppm})$ referenced to residual chloroform- $d_{1}\left(\mathrm{CDCl}_{3}\right.$, $7.27 \mathrm{ppm}), \mathrm{DMSO}-d_{6}\left(\left(\mathrm{CD}_{3}\right)_{2} \mathrm{SO}, 2.50 \mathrm{ppm}\right), \mathrm{D}_{2} \mathrm{O}(4.79 \mathrm{ppm})$, methanol- $d_{4}\left(\mathrm{CD}_{3} \mathrm{OD}, 3.31 \mathrm{ppm}\right)$ or acetonitrile- $d_{3}$ $\left(\mathrm{CD}_{3} \mathrm{CN}, 1.94 \mathrm{ppm}\right)$. Coupling constants are expressed in Hertz $(\mathrm{Hz})$. The following abbreviations are used to express the multiplicity: s (singlet), d (doublet), t (triplet), q (quadruplet), quint (quintuplet), $h$ (hextuplet), hept (heptuplet), $\mathrm{m}$ (multiplet), br (broad signal). ${ }^{13} \mathrm{C}-\mathrm{NMR}$ spectra were recorded on the same instrument at 100.6 MHz. The chemical shifts are expressed in parts per million (ppm), reported from the central of chloroform- $d_{1}\left(\mathrm{CDCl}_{3}, 77.16 \mathrm{ppm}\right), \mathrm{DMSO}-d_{6}\left(\left(\mathrm{CD}_{3}\right)_{2} \mathrm{SO}, 39.52 \mathrm{ppm}\right)$, methanol- $d_{4}\left(\mathrm{CD}_{3} \mathrm{OD}, 49.00 \mathrm{ppm}\right)$ or acetonitrile- $d_{3}\left(\mathrm{CD}_{3} \mathrm{CN}, 118.26,1.32 \mathrm{ppm}\right)$. Coupling constants are expressed in $\mathrm{Hertz}(\mathrm{Hz})$. The following abbreviations are used to express the multiplicity: $s$ (singlet), $d$ (doublet), $t$ (triplet). ${ }^{31} \mathrm{P}-\mathrm{NMR}$ spectra were recorded on the same instrument at $161.99 \mathrm{MHz}$. The chemical shifts are expressed in parts per million (ppm), reported from $\mathrm{H}_{3} \mathrm{PO}_{4}$ 85\%. The following abbreviations are used to express the multiplicity: $\mathrm{s}$ (singlet), $\mathrm{d}$ (doublet). Mass Spectra (MS): For low and high resolution spectra, the spectrometers used were electrospray ionization (ESI) WATERS Micromass Q-Tof spectrometer with as internal reference $\mathrm{H}_{3} \mathrm{PO}_{4}(0.1 \%$ in water/ acetonitrile, $1: 1)$. Chromatography: Thin Layer Chromatography (TLC) was performed on precoated plates of silica gel 60 F254 Merck. Visualisation was performed with UV light followed by phosphomolybdic acid solution or permanganate solution followed by heating as developing agents. Flash Chromatography was performed manually with silica gel $60 \AA 35-70 \mu \mathrm{m}$ SDS or using a CombiFlash Companion/TS with prepacked column (4 to $120 \mathrm{~g}$ scale) with 35-70 $\mu \mathrm{m}$. Purification of solvents and reagents: Solvent were purified before using by classical techniques under nitrogen: Tetrahydrofuran, diethyl ether, toluene, dichloromethane and acetonitrile were obtained from a dry solvent dispenser. Dimethylformamide was dried over activated $3 \AA$ molecular sieves. All air and/or water sensitive reactions were carried out under nitrogen atmosphere with dry, freshly distilled solvents and reagents when possible. All corresponding glassware was carefully dried under vacuum with a flameless heat gun.

\section{General procedure. Synthesis of tetraethyl methylene bis(phosphonate) (BPs) derivatives}

Tetraethyl methylenebis(phosphonate) (1). First method. In a dry, and nitrogen-flushed $500 \mathrm{~mL}$ threenecked, round-bottom flask equipped with a Dean Stark and a condenser were charged triethyl phosphite (147.62 mL, $0.86 \mathrm{~mol}, 6 \mathrm{eq}$ ) and dibromoethane $(10 \mathrm{~mL}, 0.14 \mathrm{~mol}, 1 \mathrm{eq})$. The mixture was heated at $150{ }^{\circ} \mathrm{C}$ under stirring for 48 hours. A vacuum distillation using Kugelrhor apparatus was used to purify the product $\left(40 \times 10^{-3} \mathrm{mbar}, 100^{\circ} \mathrm{C}\right)$. A colorless oil was obtained (11 g, yield $\left.27 \%\right)$.

Second method. In a dry, and nitrogen-flushed $500 \mathrm{~mL}$ three-necked round-bottom flask equipped with a condenser was charged diethyl phosphite $(80 \mathrm{~g}, 0.57 \mathrm{~mol}, 3 \mathrm{eq})$ followed by step-wise addition of portions of sodium metal ( $6.5 \mathrm{~g}, 0.28 \mathrm{~mol}, 1.47 \mathrm{eq})$ while stirring. Dichloromethane (16 g, $0.19 \mathrm{~mol}, 1 \mathrm{eq})$ was then added dropwise (initially $10 \mathrm{~g}$ followed by $6 \mathrm{~g}, 1 \mathrm{~h}$ and $30 \mathrm{~min}$ later), and the mixture was stirred at room temperature for $53 \mathrm{~h}$. Hexane $(100 \mathrm{~mL})$ was added, the mixture was filtered and the filtrate solvent was evaporated. After vacuum distillation to remove impurities, the pure product was obtained from distillation 
$94^{\circ} \mathrm{C}\left(70 \times 10^{-3} \mathrm{mbar}\right)$ at $102^{\circ} \mathrm{C}\left(20 \times 10^{-3} \mathrm{mbar}\right)$ as a colorless oil (5.34 g, yield $\left.10 \%\right) .{ }^{31} \mathrm{P}-\mathrm{NMR}(162 \mathrm{MHz}, \mathrm{DMSO}) \delta$ 19.44 (s). ${ }^{1} \mathrm{H}-\mathrm{NMR}\left(400 \mathrm{MHz}, \mathrm{CDCl}_{3}\right) \delta 4.19-4.11\left(\mathrm{~m}, 8 \mathrm{H}, 4 \mathrm{OCH}_{2} \mathrm{CH}_{3}\right), 2.41\left(\mathrm{t}, J 21.0 \mathrm{~Hz}, 2 \mathrm{H}, \mathrm{PC} \underline{H}_{2} \mathrm{P}\right), 1.32(\mathrm{t}, J$ $\left.7.1 \mathrm{~Hz}, 12 \mathrm{H}, 4 \mathrm{OCH}_{2} \mathrm{CH}_{3}\right) .{ }^{13} \mathrm{C}-\mathrm{NMR}\left(101 \mathrm{MHz}^{\mathrm{C} C D C l}\right)_{3} \delta 62.72-62.57\left(\mathrm{~m}, \mathrm{C}_{2}\right), 25.45\left(\mathrm{t}, \mathrm{J} 136.8 \mathrm{~Hz}, \mathrm{C}_{1}\right), 16.42(\mathrm{~m}$, $\mathrm{C}_{3}$ ).

Tetraethyl propane-2,2-diylbis(phosphonate) (3a). In a dry, and nitrogen-flushed three-necked round-bottom flask equipped with a condenser were charged dry benzene $(5 \mathrm{~mL})$, tetraethyl methylenebisphosphonate $(2.5 \mathrm{~g}$, $8.67 \mathrm{mmol}, 1 \mathrm{eq})$, methyl iodide $(5.41 \mathrm{~g}, 38.16 \mathrm{mmol}, 4.4 \mathrm{eq})$ and tBuOK $(2.14 \mathrm{~g}, 19.80 \mathrm{mmol}$, 2.2eq). The mixture was stirred at room temperature for one day. The solvent was subsequently removed under vacuum. After treatment with distilled water, extraction with dichloromethane, and drying over $\mathrm{MgSO}_{4}$ followed by filtration and evaporation of the solvent, a colorless oil was obtained (2.71g, yield 99\%). ${ }^{31} \mathrm{P}-\mathrm{NMR}\left(162 \mathrm{MHz}, \mathrm{CDCl}_{3}\right) \delta$ 27.59 (s). ${ }^{1} \mathrm{H}-\mathrm{NMR}\left(400 \mathrm{MHz}, \mathrm{CDCl}_{3}\right) \delta 4.21-4.14\left(\mathrm{~m}, 8 \mathrm{H}, \mathrm{OC}_{\mathrm{H}_{2}} \mathrm{CH}_{3}\right), 1.42\left(\mathrm{t}, \mathrm{J} 16.3 \mathrm{~Hz}, 6 \mathrm{H}, \mathrm{C}\left(\mathrm{CH}_{3}\right)_{2}\right), 1.32(\mathrm{t}, \mathrm{J}$ $\left.7.1 \mathrm{~Hz}, 12 \mathrm{H}, \mathrm{OCH}_{2} \mathrm{CH}_{3}\right) .{ }^{13} \mathrm{C}-\mathrm{NMR}\left(101 \mathrm{MHz}^{\mathrm{C} C \mathrm{CD}_{3}}\right) \delta 62.78-62.59\left(\mathrm{~m}, \mathrm{C}_{2}\right), 36.70\left(\mathrm{t}, \mathrm{J} 136.6 \mathrm{~Hz}, \mathrm{C}_{1}\right), 19.56(\mathrm{t}, \mathrm{J}$ $\left.5.6 \mathrm{~Hz}, \mathrm{C}_{4}\right), 16.61-16.43\left(\mathrm{~m}, \mathrm{C}_{3}\right)$.

Tetraethyl pentane-3,3-diylbis(phosphonate) (3b). In a dry and nitrogen-flushed three-necked equipped with a condenser were charged dry benzene $(5 \mathrm{~mL})$, tetraethylmethylene bisphosphonate $(1.8 \mathrm{~g}, 6.24 \mathrm{mmol}, 1 \mathrm{eq})$, ethyl bromide $(3 \mathrm{~g}, 27.47 \mathrm{mmol}, 4.4 \mathrm{eq})$ and sodium hydride $60 \%$ in oil $(0.33 \mathrm{~g}, 13.73 \mathrm{mmol}, 2.2 \mathrm{eq})$. The mixture was stirred at room temperature for one day. The solvent was then evaporated. After treatment with distilled water, extraction with dichloromethane, drying over $\mathrm{MgSO}_{4}$ followed by filtration and evaporation of the solvent, a colorless oil was obtained (2.1g, yield 98\%). ${ }^{31} \mathrm{P}-\mathrm{NMR}\left(162 \mathrm{MHz}, \mathrm{CDCl}_{3}\right) \delta 27.14$ (s). ${ }^{1} \mathrm{H}-\mathrm{NMR}(400$ $\left.\mathrm{MHz}, \mathrm{CDCl}_{3}\right) \delta 4.14-4.06\left(\mathrm{~m}, 8 \mathrm{H}, \mathrm{OC}_{2} \mathrm{CH}_{3}\right), 1.96-1.85\left(\mathrm{~m}, 4 \mathrm{H}, \mathrm{C}\left(\mathrm{C}_{2} \mathrm{CH}_{3}\right)_{2}\right), 1.25\left(\mathrm{t}, \mathrm{J} 7.1 \mathrm{~Hz}, 12 \mathrm{H}, \mathrm{OCH}_{2} \underline{\mathrm{C}}_{3}\right)$, $0.98\left(\mathrm{t}, \mathrm{J} 7.5 \mathrm{~Hz}, 6 \mathrm{H}, \mathrm{C}\left(\mathrm{CH}_{2} \mathrm{CH}_{3}\right)_{2}\right) .{ }^{13} \mathrm{C}-\mathrm{NMR}\left(101 \mathrm{MHz}, \mathrm{CDCl}_{3}\right) \delta 62.31-62.17\left(\mathrm{~m}, \mathrm{C}_{2}\right), 46.07\left(\mathrm{t}, \mathrm{J} 130.2 \mathrm{~Hz}, \mathrm{C}_{1}\right)$, $22.77\left(t, J 4.7 \mathrm{~Hz}, \mathrm{C}_{4}\right), 16.51-16.38\left(\mathrm{~m}, \mathrm{C}_{3}\right), 9.24\left(\mathrm{t}, \mathrm{J}, 7.2 \mathrm{~Hz}, \mathrm{C}_{5}\right)$.

Tetraethyl (2-methoxyethane-1,1-diyl)bis(phosphonate) (4). Step 1. In a three-necked flask containing 250 $\mathrm{mL}$ of $\mathrm{MeOH}$, extra dry, anhydrous diethylamine $(9.05 \mathrm{ml}, 86.74 \mathrm{mmol}, 1 \mathrm{eq})$ and paraformaldehyde (13.02 g, $433.70 \mathrm{mmol}, 5 \mathrm{eq}$ ) were introduced. The mixture was then heated until a clear and homogeneous solution was obtained ( 10h at reflux), and then tetraethyl methylenebisphosphonate $(21.55 \mathrm{~mL}, 86.74 \mathrm{mmol}, 1 \mathrm{eq})$ was added and mixture stirred while refluxing for $20 \mathrm{~h}$. The mixture was concentrated in vacuo, taken up in methanol, concentrated again to expel paraformaldehyde and excess diethylamine, then taken up in toluene and concentrated to remove traces of $\mathrm{MeOH}$. After evaporation of solvent, we obtained $26.98 \mathrm{~g}$ of yellow oil (94\% yield). ${ }^{31} \mathrm{P}-\mathrm{NMR}\left(162 \mathrm{MHz}, \mathrm{CDCl}_{3}\right) \delta 21.20$ (s). ${ }^{1} \mathrm{H}-\mathrm{NMR}\left(400 \mathrm{MHz}, \mathrm{CDCl}_{3}\right) \delta 4.23-4.07\left(\mathrm{~m}, 8 \mathrm{H}, \mathrm{OCH}_{2} \mathrm{CH}_{3}\right)$, 3.85 (td, J 16.2, $5.5 \mathrm{~Hz}, 2 \mathrm{H}, \mathrm{CHCH}_{2} \mathrm{OCH}_{3}$ ), 3.33 (s, 3H, $\mathrm{CHCH}_{2} \mathrm{OCH}_{3}$ ), 2.65 (tt, J 23.8, 5.5 Hz, 1H, $\mathrm{CHCH}_{2} \mathrm{OCH}_{3}$ ), $1.30\left(\mathrm{t}, \mathrm{J} 7.1 \mathrm{~Hz}, 12 \mathrm{H}, \mathrm{OCH}_{2} \mathrm{CH}_{3}\right) .{ }^{13} \mathrm{C}-\mathrm{NMR}\left(101 \mathrm{MHz}^{\mathrm{CCDCl}}\right)_{3} \delta 67.94$ (t, J 4.5 Hz, $\left.\mathrm{C}_{4}\right), 62.46$ (dd, J $6.8 \mathrm{~Hz}, \mathrm{C}_{2}$ ), $58.55\left(\mathrm{~s}, \mathrm{C}_{5}\right), 38.65$ (t, J $\left.132.6 \mathrm{~Hz}, \mathrm{C}_{1}\right), 16.19$ (dd, J $\left.6.7 \mathrm{~Hz}, \mathrm{C}_{3}\right)$. HRMS (TOF MS ES $\left.{ }^{+}\right)(\mathrm{m} / \mathrm{z}):[\mathrm{M}+\mathrm{H}]^{+}$calcd for $\mathrm{C}_{11} \mathrm{H}_{27} \mathrm{O}_{7} \mathrm{P}$ 333.1232. Found: 333.1232.

Tetraethyl ethene-1,1-diylbis(phosphonate) (5). Step 2. The yellow oil obtained previously was again taken up in toluene and decanted into a Dean-Stark assembly. A catalytic amount of $p$-toluenesulfonic acid monohydrate $(0.8 \mathrm{~g}, 4.2 \mathrm{mmol})$ was added and the mixture was refluxed for $18 \mathrm{~h}$. The mixture was then concentrated under vacuum, taken up in dichloromethane and washed twice with water. The organic fractions were combined and dried over anhydrous $\mathrm{Mg}_{2} \mathrm{SO}_{4}$ and then concentrated in vacuum. The distillation under pressure $\left(83^{\circ} \mathrm{C}, 0.02 \mathrm{Torr}\right)$ of the yellow oil gave 12.76 (47\% yield) of the desired tetraethyl vinylbisphosphonate as a colorless oil. ${ }^{31} \mathrm{P}-\mathrm{NMR}\left(162 \mathrm{MHz}, \mathrm{CDCl}_{3}\right) \delta 13.00(\mathrm{~s}) .{ }^{1} \mathrm{H}-\mathrm{NMR}\left(400 \mathrm{MHz}, \mathrm{CDCl}_{3}\right) \delta 6.92$ (dd, $\left.2 \mathrm{H}, \mathrm{J} 37.8 \mathrm{~Hz}, \underline{\mathrm{H}}_{2} \mathrm{C}=\mathrm{C}\right), 4.19-3.91\left(\mathrm{~m}, 8 \mathrm{H}, \mathrm{OC}_{2} \mathrm{CH}_{3}\right), 1.28\left(\mathrm{t}, J 7.1 \mathrm{~Hz}, 12 \mathrm{H}, \mathrm{OCH}_{2} \underline{\mathrm{C}}_{3}\right) .{ }^{13} \mathrm{C}-\mathrm{NMR}(101 \mathrm{MHz}$, $\left.\mathrm{CDCl}_{3}\right) \delta 148.76\left(\mathrm{~s}, \mathrm{C}_{4}\right), 131.93\left(\mathrm{t}, J 166.4 \mathrm{~Hz}, \mathrm{C}_{1}\right.$ ), $62.38\left(\mathrm{t}, J 2.8 \mathrm{~Hz}, \mathrm{C}_{2}\right.$ ), 16.01 (t, J $\left.6.5 \mathrm{~Hz}, \mathrm{C}_{3}\right)$. HRMS (TOF MS $\left.\mathrm{ES}^{+}\right)(\mathrm{m} / \mathrm{z}):[\mathrm{M}+\mathrm{H}]^{+}$calcd for $\mathrm{C}_{11} \mathrm{H}_{23} \mathrm{O}_{6} \mathrm{P}_{2}$ 301.0970. Found: 301.0969 . 
Tetraethyl ethane-1,1-diylbis(phosphonate) (2a). In a two-necked flask under nitrogen containing $210 \mathrm{~mL}$ of ethyl acetate were added vinylbisphosphonate $(12.46 \mathrm{~g}, 41.50 \mathrm{mmol}, 1 \mathrm{eq})$ and palladium on charcoal $10 \%$ $(1.87 \mathrm{~g}, 17.43 \mathrm{mmol})$. Through a septum was added hydrogen at atmospheric pressure. After 72 hours of stirring, the mixture was filtered, and the solvent evaporated, to give $12.04 \mathrm{~g}$ ( $96 \%$ yield) of a colorless oil. ${ }^{31} \mathrm{P}$ NMR (162 MHz, CDCl $\left.{ }_{3}\right) \delta 24.01$ (s). ${ }^{1} \mathrm{H}-\mathrm{NMR}\left(400 \mathrm{MHz}, \mathrm{CDCl}_{3}\right) \delta 4.20-4.12\left(\mathrm{~m}, 8 \mathrm{H}, \mathrm{OC}_{2} \mathrm{CH}_{3}\right), 2.35$ (tq, J 23.6 , $\left.7.4 \mathrm{~Hz}, 1 \mathrm{H}, \mathrm{PC} \underline{\mathrm{H}}\left(\mathrm{CH}_{3}\right) \mathrm{P}\right), 1.44$ (td, J 17.2, 7.4 Hz, 1H, PCH(C $\left.\left.\underline{\mathrm{H}}_{3}\right) \mathrm{P}\right), 1.32\left(\mathrm{t}, J 7.1 \mathrm{~Hz}, 1 \mathrm{H}, \mathrm{OCH}_{2} \mathrm{CH}_{3}\right) .{ }^{13} \mathrm{C}-\mathrm{NMR}(101$ $\mathrm{MHz}, \mathrm{CDCl}_{3}$ ) $\delta 62.21\left(\mathrm{dd}, J\right.$ 6.8, $6.8 \mathrm{~Hz}, \mathrm{C}_{2}$ ), 30.88 (t, J $136.1 \mathrm{~Hz}, \mathrm{C}_{1}$ ), 16.09 (dd, J 6.3, $\left.1.8 \mathrm{~Hz}, \mathrm{C}_{3}\right), 9.97$ (t, J $6.2 \mathrm{~Hz}$, $\mathrm{C}_{4}$ ). HRMS (TOF MS ES ${ }^{+}$) (m/z): $[\mathrm{M}+\mathrm{H}]^{+}$calcd for $\mathrm{C}_{10} \mathrm{H}_{25} \mathrm{O}_{6} \mathrm{P}_{2}$ 303.1126. Found: 303.1127 .

\section{General procedure for substitution on the bisphosphonate's methylene bridge}

In a dry and nitrogen-flushed three-necked flask equipped with a condenser were charged dry tetrahydrofuran, tetraethyl methylenebisphosphonate, alkyl halide derivatives and sodium hydride $60 \%$ in oil. The mixture was stirred at room temperature or heated two days at $50{ }^{\circ} \mathrm{C}$ depending on the nature of the electrophile. The solvent was then concentrated under vacuum. The residue was treated with distilled water and extracted with dichloromethane. The organic layers were combined, dried over $\mathrm{MgSO}_{4}$ and concentrated after filtration. The crude was purified by chromatography on silica gel.

Tetraethyl (9-bromononane-1,1-diyl)bis(phosphonate) (2c). Tetraethyl methylenebisphosphonate (1 g, 3.48 mmol, 1 eq), 1,8-dibromooctane (1.89 g, $6.96 \mathrm{mmol}, 2 \mathrm{eq})$, sodium hydride 60\% in oil $(0.153 \mathrm{~g}, 3.82 \mathrm{mmol}, 1.1$ eq). After separation on silica gel (AcOEt / MeOH 7:3) a colorless oil was obtained ( $0.20 \mathrm{~g}$, yield $12 \%) .{ }^{31} \mathrm{P}-\mathrm{NMR}$ $\left(162 \mathrm{MHz}, \mathrm{CDCl}_{3}\right) \delta 24.14$ (s). ${ }^{1} \mathrm{H}-\mathrm{NMR}\left(400 \mathrm{MHz}, \mathrm{CDCl}_{3}\right) \delta 4.21-4.12\left(\mathrm{~m}, 8 \mathrm{H}, 4 \mathrm{OCH}_{2} \mathrm{CH}_{3}\right), 3.40(\mathrm{t}, \mathrm{J} 6.9 \mathrm{~Hz}, 2 \mathrm{H}$, $\left.\mathrm{C}_{2} \mathrm{Br}\right), 2.25$ (tt, J 24.1, $\left.6.0 \mathrm{~Hz}, 1 \mathrm{H}, \mathrm{PC} \underline{\mathrm{HP}}\right), 1.93-1.73\left(\mathrm{~m}, 4 \mathrm{H}, \mathrm{PCH}\left(\underline{\mathrm{C}}_{2}\right) \mathrm{P}, \mathrm{C}_{2} \mathrm{CH}_{2} \mathrm{Br}\right), 1.60-1.35(\mathrm{~m}, 22 \mathrm{H}$, $40 \mathrm{OH}_{2} \mathrm{CH}_{3}, \mathrm{PCHPCH}{ }_{2} \underline{\mathrm{C}}_{2} \mathrm{C}_{2}{ }_{2} \underline{\mathrm{C}}_{2} \mathrm{C}_{2} \mathrm{C}_{2} \mathrm{C}_{2} \mathrm{CH}_{2} \mathrm{CH}_{2} \mathrm{Br}$ ). ${ }^{13} \mathrm{C}-\mathrm{NMR}\left(101 \mathrm{MHz}, \mathrm{CDCl}_{3}\right) \delta 62.46$ (dd, J 16.0, 6.7 Hz, C2), 36.77 (t, J $134.6 \mathrm{~Hz}, \mathrm{C}_{1}$ ), 29.17 (d, J $19.3 \mathrm{~Hz}, \mathrm{C}_{10}, \mathrm{C}_{11}$ ), 28.42 (d, J $56.3 \mathrm{~Hz}, \mathrm{C}_{6}, \mathrm{C}_{7}, \mathrm{C}_{8}, \mathrm{C}_{9}$ ), 25.55 (t, J 5.0 Hz, C, $\mathrm{C}_{5}$ ), $16.45\left(\mathrm{dd}, \mathrm{J}\right.$ 6.3, $\left.2.5 \mathrm{~Hz}, \mathrm{C}_{3}\right)$. HRMS (TOF MS ES $\left.{ }^{+}\right)(\mathrm{m} / \mathrm{z}):[\mathrm{M}+\mathrm{H}]^{+}$calcd for $\mathrm{C}_{17} \mathrm{H}_{38} \mathrm{O}_{6} \mathrm{P} 2 \mathrm{Br}$ 479.1327. Found: 479.1328

Tetraethyl nonane-1,1-diylbis(phosphonate) (2d). Tetraethylmethylene bisphosphonate (4.31 mL, 17.34 $\mathrm{mmol}, 1 \mathrm{eq}), 1$-bromooctane $(6.03 \mathrm{~mL}, 34.69 \mathrm{mmol}, 2 \mathrm{eq})$, sodium hydride $60 \%$ in oil $(1.04 \mathrm{~g}, 26.02 \mathrm{mmol}, 1.5$ eq). After separation on silica gel (AcOEt / MeOH 9:1), a colorless oil was obtained (1.92 g, yield $28 \%$ ). ${ }^{31} \mathrm{P}-\mathrm{NMR}$ $\left(162 \mathrm{MHz}, \mathrm{CDCl}_{3}\right) \delta 23.96$ (s). ${ }^{1} \mathrm{H}-\mathrm{NMR}\left(400 \mathrm{MHz}, \mathrm{CDCl}_{3}\right) \delta 4.03-3.90\left(\mathrm{~m}, 8 \mathrm{H}, \mathrm{C}_{2} \mathrm{CH}_{3}\right), 2.08(\mathrm{tt}, J 24.1,6.0 \mathrm{~Hz}$, $1 \mathrm{H}, \mathrm{PC} \underline{\mathrm{HP}}), 1.82-1.69\left(\mathrm{~m}, 2 \mathrm{H},-\mathrm{CH}_{2} \mathrm{CH}_{2} \mathrm{CH}_{3}\right), 1.44-1.26\left(\mathrm{~m}, 2 \mathrm{H}, \mathrm{PCHPCH}_{2} \mathrm{CH}_{2} \mathrm{CH}_{2} \mathrm{CH}_{2} \mathrm{CH}_{2} \mathrm{CH}_{2} \mathrm{CH}_{2} \mathrm{CH}_{2} \mathrm{CH}_{3}\right), 1.17-$ $1.07\left(\mathrm{~m}, 22 \mathrm{H}, \mathrm{OCH}_{2} \mathrm{CH}_{3}, \mathrm{PCHPCH}_{2} \mathrm{CH}_{2} \mathrm{CH}_{2} \mathrm{CH}_{2} \mathrm{CH}_{2} \mathrm{CH}_{2} \mathrm{CH}_{2} \mathrm{CH}_{2} \mathrm{CH}_{3}\right), 0.68\left(\mathrm{t}, J 6.9 \mathrm{~Hz}, 3 \mathrm{H},-\mathrm{CH}_{2} \mathrm{CH}_{2} \mathrm{CH}_{2} \mathrm{CH}_{3}\right) .{ }^{13} \mathrm{C}_{-}$

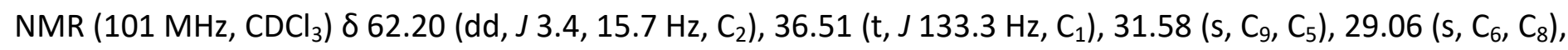
$28.94\left(\mathrm{~d}, J 0.7 \mathrm{~Hz}, \mathrm{C}_{7}\right.$ ), 28.84 (t, J $6.5 \mathrm{~Hz}, \mathrm{C} 11$ ), 25.29 (t, J $5.1 \mathrm{~Hz}, \mathrm{C}_{10}$ ), $22.38\left(\mathrm{~s}, \mathrm{C}_{4}\right.$ ), 16.14 (dd, J 6.4, $2.7 \mathrm{~Hz}, \mathrm{C}_{3}$ ), $13.81\left(\mathrm{~s}, \mathrm{C}_{11}\right)$. HRMS (TOF MS ES ${ }^{+}$) (m/z): $[\mathrm{M}+\mathrm{H}]^{+}$calcd for $\mathrm{C}_{17} \mathrm{H}_{39} \mathrm{O}_{6} \mathrm{P}_{2}$ 401.2222. Found: 401.2219.

Tetraethyl (2-(p-tolyl)ethane-1,1-diyl)bis(phosphonate) (2e). Tetraethylmethylene bisphosphonate $(4.31 \mathrm{~mL}$, $17.34 \mathrm{mmol}, 1 \mathrm{eq}$ ), 4-methylbenzyl bromide (4.81 g, $26.02 \mathrm{mmol}, 1.5 \mathrm{eq})$, sodium hydride $60 \%$ (0.83 g, 20.81 $\mathrm{mmol}, 1.3 \mathrm{eq}$ ). After separation on silica gel (AcOEt / MeOH 9.5:0.5), the mono derivative was obtained as a pale yellow oil (3.59 g, yield 53\%,) and the di derivative (3e) as a pale yellow oil $(0.19 \mathrm{~g}$, yield $2.21 \%) .{ }^{31} \mathrm{P}-\mathrm{NMR}$

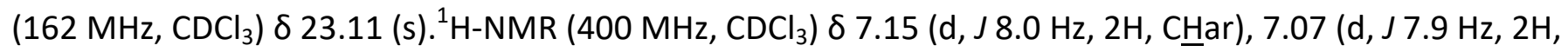
C블), $4.18-4.01\left(\mathrm{~m}, 8 \mathrm{H}, \mathrm{OCH}_{2} \mathrm{CH}_{3}\right), 3.20$ (td, J 16.5, $\left.6.2 \mathrm{~Hz}, 2 \mathrm{H}, \mathrm{P}-\mathrm{CH}\left(\mathrm{CH}_{2}\right)-\mathrm{P}\right), 2.62(\mathrm{tt}, J 23.9,6.2 \mathrm{~Hz}, 1 \mathrm{H}$, PC바), $2.30\left(\mathrm{~s}, 3 \mathrm{H}, \mathrm{CH}_{2}-\mathrm{Ar}-\mathrm{C}_{3}\right), 1.26\left(\mathrm{q}, J 7.1 \mathrm{~Hz}, 12 \mathrm{H}, \mathrm{OCH}_{2} \mathrm{C}_{3}\right) .{ }^{13} \mathrm{C}-\mathrm{NMR}\left(101 \mathrm{MHz}, \mathrm{CDCl}_{3}\right) \delta 136.34(\mathrm{t}, J 7.4$ $\mathrm{Hz}, \mathrm{C}_{5}$ ), $135.73\left(\mathrm{~s}, \mathrm{C}_{8}\right), 128.63$ (d, J $12.2 \mathrm{~Hz}, \mathrm{C}_{6}, \mathrm{C}_{7}$ ), 62.32 (dd, J 6.7, $17.9 \mathrm{~Hz}, \mathrm{C}_{2}$ ), 38.93 (t, J 132.2 Hz, $\mathrm{C}_{1}$ ), 30.57 $\left(\mathrm{t}, J 7.8 \mathrm{~Hz}, \mathrm{C}_{4}\right), 20.81\left(\mathrm{~s}, \mathrm{C}_{9}\right), 16.10\left(\mathrm{~d}, J 3.4 \mathrm{~Hz}, \mathrm{C}_{3}\right)$. HRMS (TOF MS ES $\left.{ }^{+}\right)(\mathrm{m} / \mathrm{z}):[\mathrm{M}+\mathrm{H}]^{+}$calcd for $\mathrm{C}_{17} \mathrm{H}_{31} \mathrm{O}_{6} \mathrm{P}_{2}$ 393.1596. Found: 393.1598. 
Tetraethyl (1,3-di-p-tolylpropane-2,2-diyl)bis(phosphonate) (3e). ${ }^{31} \mathrm{P}-\mathrm{NMR}\left(162 \mathrm{MHz}, \mathrm{CDCl}_{3}\right) \delta 24.49$ (s). ${ }^{1} \mathrm{H}-$

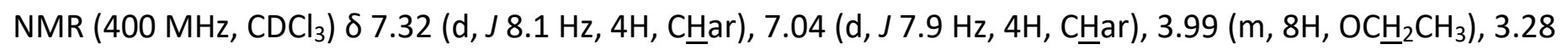
$\left(\mathrm{t}, J 16.1 \mathrm{~Hz}, 4 \mathrm{H}, \mathrm{P}-\mathrm{C}\left(\mathrm{C}_{2}\right)_{2}-\mathrm{P}\right), 2.30\left(\mathrm{~s}, 6 \mathrm{H}, \mathrm{CH}_{2}-\mathrm{Ar}_{-} \underline{\mathrm{C}}_{3}\right), 1.13\left(\mathrm{t}, J 7.1 \mathrm{~Hz}, 12 \mathrm{H}, \mathrm{OCH}_{2} \mathrm{CH}_{3}\right) .{ }^{13} \mathrm{C}-\mathrm{NMR}(101 \mathrm{MHz}$, $\mathrm{CDCl}_{3}$ ) $\delta 136.09\left(\mathrm{~s}, \mathrm{C}_{8}\right), 133.69$ (t, J $6.7 \mathrm{~Hz}, \mathrm{C}_{5}$ ), $131.72\left(\mathrm{~s}, \mathrm{C}_{6}\right), 128.13\left(\mathrm{~s}, \mathrm{C}_{7}\right), 62.30$ (dd, J 5.3, $12 \mathrm{~Hz}, \mathrm{C}_{2}$ ), 48.94 (t, J $131.2 \mathrm{~Hz}, \mathrm{C}_{1}$ ), 37.36 (t, J 4.6 Hz, $\mathrm{C}_{4}$ ), $21.14\left(\mathrm{~s}, \mathrm{C}_{9}\right), 16.22$ (dd, J 5.1, 12.3 Hz, $\left.\mathrm{C}_{3}\right)$. HRMS (TOF MS ES ${ }^{+}$(m/z) : $[\mathrm{M}+\mathrm{H}]^{+}$calcd for $\mathrm{C}_{25} \mathrm{H}_{39} \mathrm{O}_{6} \mathrm{P}_{2}$ 497.2222. Found: 497.2223.

Tetraethyl (2-(4-(bromomethyl)phenyl)ethane-1,1-diyl)bis(phosphonate) (2f). Tetraethylmethylene bisphosphonate (1 g, $3.48 \mathrm{mmol}, 1 \mathrm{eq}), 1$,4-bis(bromomethyl)benzene (1.83 g, $6.96 \mathrm{mmol}, 2 \mathrm{eq})$, sodium hydride $60 \%$ ( $0.208 \mathrm{~g}, 5.22 \mathrm{mmol}, 1.5 \mathrm{eq})$. After separation on silica gel (AcOEt / MeOH 9.5:0.5), the mono derivative was obtained as a yellow oil $(0.40 \mathrm{~g}$, yield $25 \%$, ) and the di derivative (3f) as a yellow oil $(0.12 \mathrm{~g}$, yield 5\%). ${ }^{31} \mathrm{P}-\mathrm{NMR}\left(162 \mathrm{MHz}, \mathrm{CDCl}_{3}\right) \delta 22.76$ (s). ${ }^{1} \mathrm{H}-\mathrm{NMR}\left(400 \mathrm{MHz}, \mathrm{CDCl}_{3}\right) \delta 7.30(\mathrm{~d}, J 8.1 \mathrm{~Hz}, 2 \mathrm{H}, \mathrm{C} \underline{\mathrm{Har}}), 7.25$

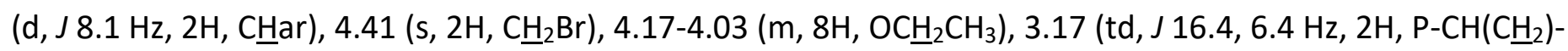
P), 2.62 (tt, J 23.7, $6.4 \mathrm{~Hz}, 1 \mathrm{H}, \mathrm{PC} \underline{\mathrm{H}}$ ), 1.25 (td, J 7.0 Hz, 12H, OCH $\left.\mathrm{CH}_{3}\right) .{ }^{13} \mathrm{C}-\mathrm{NMR}\left(101 \mathrm{MHz}, \mathrm{CDCl}_{3}\right) \delta 140.16(\mathrm{~s}$, $\left.\mathrm{C}_{5}\right), 136.20\left(\mathrm{~s}, \mathrm{C}_{8}\right), 129.55\left(\mathrm{~s}, \mathrm{C}_{6}\right), 129.11\left(\mathrm{~s}, \mathrm{C}_{7}\right), 62.68\left(\mathrm{dd}, J\right.$ 22.42, $\left.6.7 \mathrm{~Hz}, \mathrm{C}_{2}\right), 39.10$ (t, J $\left.132.9 \mathrm{~Hz}, \mathrm{C}_{1}\right), 33.56$ (s, $\mathrm{C}_{9}$ ), 31.15 (t, J $4.9 \mathrm{~Hz}, \mathrm{C}_{4}$ ), 16.33 (dd, J $6.6 \mathrm{~Hz}, \mathrm{C}_{3}$ ). HRMS (TOF MS ES ${ }^{+}$(m/z) : $[\mathrm{M}+\mathrm{H}]^{+}$calcd for $\mathrm{C}_{17} \mathrm{H}_{30} \mathrm{O}_{6} \mathrm{P}_{2} \mathrm{Br}$ 471.0701. Found: 471.0704.

Tetraethyl (1,3-bis(4-(bromomethyl)phenyl)propane-2,2-diyl)bis(phosphonate) (3f). ${ }^{31} \mathrm{P}-\mathrm{NMR}$ (162 MHz, $\mathrm{CDCl}_{3}$ ) $\delta 23.85$ (s). ${ }^{1} \mathrm{H}-\mathrm{NMR}\left(400 \mathrm{MHz}, \mathrm{CDCl}_{3}\right) \delta 7.40$ (d, J $8.2 \mathrm{~Hz}, 4 \mathrm{H}, \mathrm{C} \underline{\mathrm{Har}}$ ), 7.27 (d, J 8.3 Hz, 4H, Cㅡar), 4.42 (s, $\left.4 \mathrm{H}, 2 \underline{\mathrm{H}}_{2} \mathrm{Br}\right), 4.05-3.94\left(\mathrm{~m}, 8 \mathrm{H}, \mathrm{OC}_{2} \mathrm{CH}_{3}\right), 3.31$ (t, J $\left.16.1 \mathrm{~Hz}, 4 \mathrm{H}, \mathrm{P}-\mathrm{C}\left(\underline{C}_{2}\right)_{2} \mathrm{P}\right), 1.07$ (t, J 7.1 Hz, 12H, OCH $\left.\mathrm{CH}_{3}\right)$. ${ }^{13} \mathrm{C}-\mathrm{NMR}\left(101 \mathrm{MHz}, \mathrm{CDCl}_{3}\right) \delta 137.18\left(\mathrm{t}, J 6.5 \mathrm{~Hz}, \mathrm{C}_{5}\right), 136.28\left(\mathrm{~s}, \mathrm{C}_{8}\right), 132.20\left(\mathrm{~s}, \mathrm{C}_{6}\right), 128.20\left(\mathrm{~s}, \mathrm{C}_{7}\right), 62.56-62.39$ $\left(\mathrm{m}, \mathrm{C}_{2}\right), 48.65\left(\mathrm{t}, J 131.5 \mathrm{~Hz}, \mathrm{C}_{1}\right), 37.67\left(\mathrm{t}, J 4.6 \mathrm{~Hz}, \mathrm{C}_{4}\right), 33.60\left(\mathrm{~s}, \mathrm{C}_{9}\right), 16.24$ (t, J $\left.6.5 \mathrm{~Hz}, \mathrm{C}_{3}\right)$. HRMS (TOF MS ES $\left.{ }^{+}\right)$ $(\mathrm{m} / \mathrm{z}):[\mathrm{M}+\mathrm{H}]^{+}$calcd for $\mathrm{C}_{25} \mathrm{H}_{37} \mathrm{O}_{6} \mathrm{P}_{2} \mathrm{Br}_{2}$ 653.0432. Found: 653.0433 .

Tetraethyl (1-(p-tolyl)propane-2,2-diyl)bis(phosphonate) (6). Tetraethylethane-1,1-diylbisphosphonate (2 g, $6.62 \mathrm{mmol}, 1 \mathrm{eq}), 4$-methylbenzyl bromide $(2.57 \mathrm{~g}, 13.90 \mathrm{mmol}, 1.2 \mathrm{eq})$, sodium hydride $60 \%$ (0.69 g, 17.37 $\mathrm{mmol}, 1.5 \mathrm{eq})$. After extraction with $\mathrm{ACN}(100 \mathrm{~mL})$ and hexane $(3 \times 50 \mathrm{~mL})$, the product was obtained as a pale yellow oil (3.22 g, yield 69\%). ${ }^{31} \mathrm{P}-\mathrm{NMR}\left(162 \mathrm{MHz}^{\mathrm{C} C D C l}\right)_{3} \delta 26.48$ (s). ${ }^{1} \mathrm{H}-\mathrm{NMR}\left(400 \mathrm{MHz}, \mathrm{CDCl}_{3}\right) \delta 7.17$ (d, J 8.0 $\mathrm{Hz}, 2 \mathrm{H}, \mathrm{C} \underline{\mathrm{H} a r}$ ), 7.04 (d, J 7.8 Hz, 2H, CHar), $4.17-4.08\left(\mathrm{~m}, 8 \mathrm{H}, \mathrm{OC}_{2} \mathrm{CH}_{3}\right), 3.20$ (dd, J 15.6, $13.8 \mathrm{~Hz}$, $\left.2 \mathrm{H}, \mathrm{PC}\left(\mathrm{CH}_{3}\right)\left(\mathrm{C}_{2}\right) \mathrm{P}\right), 2.30\left(\mathrm{~s}, 3 \mathrm{H},-\mathrm{CH}_{2}-\mathrm{Ar}-\mathrm{CH}_{3}\right), 1.40\left(\mathrm{t}, J 16.7 \mathrm{~Hz}, 3 \mathrm{H}, \mathrm{PC}\left(\mathrm{C}_{3}\right)\left(\mathrm{CH}_{2}\right) \mathrm{P}\right), 1.25(\mathrm{dt}, J 17.4,7.1 \mathrm{~Hz}, 12 \mathrm{H}$, $\left.\mathrm{OCH}_{2} \mathrm{CH}_{3}\right) .{ }^{13} \mathrm{C}-\mathrm{NMR}\left(101 \mathrm{MHz}, \mathrm{CDCl}_{3}\right) \delta 136.11\left(\mathrm{~s}, \mathrm{C}_{8}\right), 133.02\left(\mathrm{t}, J 8.3 \mathrm{~Hz}, \mathrm{C}_{5}\right), 131.51\left(\mathrm{~s}, \mathrm{C}_{6}\right), 128.19\left(\mathrm{~s}, \mathrm{C}_{7}\right)$, $64.72-62.58\left(m, C_{2}\right), 42.38\left(t, J 133.2 \mathrm{~Hz}, \mathrm{C}_{1}\right), 37.22\left(\mathrm{t}, J 4.2 \mathrm{~Hz}, \mathrm{C}_{4}\right), 21.11\left(\mathrm{~s}, \mathrm{C}_{10}\right), 16.51-16.33\left(\mathrm{~m}, \mathrm{C}_{3}\right), 16.16$ $\left(\mathrm{t}, J 6.2 \mathrm{~Hz}, \mathrm{C}_{9}\right)$. HRMS (TOF MS ES $\left.{ }^{+}\right)(\mathrm{m} / \mathrm{z}):[\mathrm{M}+\mathrm{H}]^{+}$calcd for $\mathrm{C}_{18} \mathrm{H}_{33} \mathrm{O}_{6} \mathrm{P}_{2}$ 407.1752. Found: 407.1747.

Tetraethyl butane-2,2-diylbis(phosphonate) (7). Tetraethylethane-1,1-diylbisphosphonate (3 g, $9.93 \mathrm{mmol}, 1$ eq), 1-bromooctane ( $2.59 \mathrm{~mL}, 14.89 \mathrm{mmol}, 1.5 \mathrm{eq})$, sodium hydride 60\% (0.59 g, $14.89 \mathrm{mmol}, 1.5 \mathrm{eq})$. After separation on silica gel (AcOEt / MeOH 9.5:0.5), a colorless oil was obtained (0.10 g, yield $3 \%)$. ${ }^{31} \mathrm{P}-\mathrm{NMR}(162$ $\left.\mathrm{MHz}_{,} \mathrm{CDCl}_{3}\right) \delta 27.52$ (s). ${ }^{1} \mathrm{H}-\mathrm{NMR}\left(400 \mathrm{MHz}, \mathrm{CDCl}_{3}\right) \delta 4.18\left(\mathrm{q}, J 7.1 \mathrm{~Hz}, 8 \mathrm{H}, \mathrm{OC}_{2} \mathrm{CH}_{3}\right), 2.01-1.83\left(\mathrm{~m}, 2 \mathrm{H}, \mathrm{H}{ }_{3} \mathrm{C}-\mathrm{C}-\right.$ $\left.\mathrm{CH}_{2} \mathrm{CH}_{3}\right), 1.41\left(\mathrm{t}, J 16.6 \mathrm{~Hz}, 3 \mathrm{H}, \underline{\mathrm{H}}_{3} \mathrm{C}-\mathrm{C}-\mathrm{CH}_{2} \mathrm{CH}_{3}\right), 1.32\left(\mathrm{t}, J 7.1 \mathrm{~Hz}, 12 \mathrm{H}, \mathrm{OCH}_{2} \mathrm{C}_{3}\right), 1.08(\mathrm{t}, J 7.5 \mathrm{~Hz}, 3 \mathrm{H}, \mathrm{H} 3 \mathrm{C}-\mathrm{C}-$ $\left.\mathrm{CH}_{2} \mathrm{CH}_{3}\right) .{ }^{13} \mathrm{C}-\mathrm{NMR}\left(101 \mathrm{MHz}, \mathrm{CDCl}_{3}\right) \delta 63.07-62.10\left(\mathrm{~m}, \mathrm{C}_{2}\right), 41.09\left(\mathrm{t}, J 133.7 \mathrm{~Hz}, \mathrm{C}_{1}\right), 26.30\left(\mathrm{t}, J 4.6 \mathrm{~Hz}, \mathrm{C}_{5}\right)$, $16.61-16.42\left(m, C_{3}\right), 16.33\left(t, J 5.7 \mathrm{~Hz}, \mathrm{C}_{4}\right), 9.50\left(\mathrm{t}, J 6.4 \mathrm{~Hz}, \mathrm{C}_{6}\right)$. HRMS (TOF MS ES $\left.{ }^{+}\right)(\mathrm{m} / \mathrm{z}):[\mathrm{M}+\mathrm{H}]^{+}$calcd for $\mathrm{C}_{12} \mathrm{H}_{29} \mathrm{O}_{6} \mathrm{P}_{2}$ 331.1439. Found: 331.1441.

Tetraethyl decane-2,2-diylbis(phosphonate) (8). Tetraethylnonane-1,1-diylbisphosphonate (0.2 mL, 0.49 $\mathrm{mmol}, 1 \mathrm{eq})$, iodomethane $(0.03 \mathrm{~mL}, 0.59 \mathrm{mmol}, 1.2 \mathrm{eq})$, sodium hydride $60 \%$ in oil $(0.02 \mathrm{~g}, 0.59 \mathrm{mmol}, 1.5 \mathrm{eq})$. After extraction with DCM and water, a yellow oil was obtained ( $0.15 \mathrm{~g}$, yield $75 \%)$.

${ }^{31} \mathrm{P}-\mathrm{NMR}\left(162 \mathrm{MHz}, \mathrm{CDCl}_{3}\right) \delta 27.52(\mathrm{~s}) .{ }^{1} \mathrm{H}-\mathrm{NMR}\left(400 \mathrm{MHz}, \mathrm{CDCl}_{3}\right) \delta 4.17\left(\mathrm{~m}, 8 \mathrm{H}, \mathrm{OCH}_{2} \mathrm{CH}_{3}\right), 1.81$ (dd, J 32.9, $\left.16.5 \mathrm{~Hz}, 2 \mathrm{H}, \mathrm{PC}\left(\underline{\mathrm{C}}_{2}\right) \mathrm{P}\right), 1.54\left(\mathrm{~m}, 2 \mathrm{H}, \mathrm{PC}\left(\mathrm{CH}_{2} \underline{\mathrm{C}}_{2}\right) \mathrm{P}\right), 1.42\left(\mathrm{t}, J 16.6 \mathrm{~Hz}, 3 \mathrm{H}, \mathrm{PC}\left(\mathrm{C}_{3}\right) \mathrm{P}\right), 1.33-1.26(\mathrm{~m}, 22 \mathrm{H}$, 
$\left.\mathrm{OCH}_{2} \underline{\mathrm{CH}}_{3}, \mathrm{PCPC} \underline{\mathrm{H}}_{2} \underline{\mathrm{C}}_{2} \mathrm{CH}_{2} \mathrm{C}_{2} \mathrm{C}_{2} \mathrm{CH}_{2} \mathrm{CH}_{2} \mathrm{CH}_{2} \mathrm{CH}_{3}\right), 0.87$ (t, J $\left.6.9 \mathrm{~Hz}, 3 \mathrm{H},-\mathrm{CH}_{2} \mathrm{CH}_{3}\right) .{ }^{13} \mathrm{C}-\mathrm{NMR}\left(101 \mathrm{MHz}, \mathrm{CDCl}_{3}\right) \delta$ 62.50 (dd, J 15.6, $6.8 \mathrm{~Hz}, \mathrm{C}_{2}$ ), 40.96 (t, J $133.3 \mathrm{~Hz}, \mathrm{C}_{1}$ ), 33.35 (t, J $4.9 \mathrm{~Hz}, \mathrm{C}_{5}$ ), 31.89 (s, $\left.\mathrm{C}_{6}\right), 30.38$ (s, C 7 ), 29.30 (d, J $\left.6.6 \mathrm{~Hz}, \mathrm{C}_{8}, \mathrm{C}_{9}\right), 24.57\left(\mathrm{t}, J 5.9 \mathrm{~Hz}, \mathrm{C}_{10}\right), 22.69\left(\mathrm{~s}, \mathrm{C}_{11}\right), 16.80\left(\mathrm{t}, \mathrm{J} 5.7 \mathrm{~Hz}, \mathrm{C}_{4}\right), 16.57-16.42\left(\mathrm{~m}, \mathrm{C}_{3}\right), 14.12\left(\mathrm{~s}, \mathrm{C}_{12}\right)$. HRMS (TOF MS ES ${ }^{+}$(m/z) : [M+H] $]^{+}$calcd for $\mathrm{C}_{18} \mathrm{H}_{41} \mathrm{O}_{6} \mathrm{P}_{2}$ 415.2378. Found: 415.2374.

\section{Diethyl ((diphenylphosphoryl)methyl)phosphonate derivatives}

Diphenylphosphine oxide (10). In a dry and nitrogen-flushed three-necked flask fitted with a condenser and a dropping funnel, were charged tetrahydrofuran $(100 \mathrm{~mL})$, chlorodiphenylphosphine $(15 \mathrm{~mL}, 81.17 \mathrm{mmol}, 1 \mathrm{eq})$ at $0^{\circ} \mathrm{C}$. Water $(10 \mathrm{~mL}, 811.70 \mathrm{mmol}, 10 \mathrm{eq})$ was then added dropwise into the flask through the dropping funnel, and the reaction mixture was stirred for two hours at room temperature. The THF was evaporated and the oil obtained was diluted in DCM $(120 \mathrm{~mL})$. The solution was washed with sodium bicarbonate $(50 \mathrm{~mL})$. The organic phase was dried over magnesium sulfate, filtered and concentrated. A white solid was obtained (14.43 g, 88\%). ${ }^{31} \mathrm{P}-N M R\left(162 \mathrm{MHz}\right.$, DMSO-d 6 ) $\delta 18.52$ (s). ${ }^{31} \mathrm{P}-\mathrm{NMR}\left(162 \mathrm{MHz}, \mathrm{DMSO}-\mathrm{d}_{6}\right) \delta 18.52$ (d, J $\left.481.4 \mathrm{~Hz}\right) .{ }^{1} \mathrm{H}-$ NMR $\left(400 \mathrm{MHz}, \mathrm{CDCl}_{3}\right) \delta 8.68(\mathrm{~s}, 1 \mathrm{H}, \mathrm{P}-\underline{\mathrm{H}}), 7.75-7.36(\mathrm{~m}, 10 \mathrm{H}, \mathrm{C} \underline{\mathrm{Har}}) .{ }^{13} \mathrm{C}-\mathrm{NMR}\left(101 \mathrm{MHz}, \mathrm{CDCl}_{3}\right) \delta 132.68(\mathrm{~s}$, $C_{4}$ ), 131.50 (d, J $101.1 \mathrm{~Hz}, \mathrm{C}_{1}, \mathrm{C}_{1^{\prime}}$ ), 130.51 (d, J $11.4 \mathrm{~Hz}, \mathrm{C}_{2}, \mathrm{C}_{6}$ ), 128.80 (d, J $12.5 \mathrm{~Hz} \mathrm{C}_{3}, \mathrm{C}_{5}$ ).

Diphenylhydroxymethylphosphine oxide (11). In a dry and nitrogen-flushed two-necked flask with a condenser, were charged diphenylphosphine oxide $(4.5 \mathrm{~g}, 68.84 \mathrm{mmol}, 1 \mathrm{eq})$, para-formaldehyde $(0.86 \mathrm{~g}$, $82.61 \mathrm{mmol}, 1.2 \mathrm{eq})$ and trimethylamine $(3.90 \mathrm{~mL}, 89.49 \mathrm{mmol}, 1.3 \mathrm{eq})$. The mixture was heated at $80^{\circ} \mathrm{C}$ for five hours under stirring. Trimethylamine was evaporated and the solid was taken up in ethanol and heated for two (2) hours under stirring. After evaporation of the ethanol, a white powder was obtained (15.19 g, yield 94\%). ${ }^{31} \mathrm{P}-\mathrm{NMR}\left(162 \mathrm{MHz}, \mathrm{CDCl}_{3}\right) \delta 31.97$ (s). ${ }^{1} \mathrm{H}-\mathrm{NMR}\left(400 \mathrm{MHz}, \mathrm{CDCl}_{3}\right) \delta 7.78-7.29(\mathrm{~m}, 10 \mathrm{H}, \mathrm{C}$ Har), 6.14 (sL, $\left.1 \mathrm{H}, \mathrm{CH}_{2} \mathrm{O} \underline{\mathrm{H}}\right), 4.40$ (s, 2H, $\left.\underline{\mathrm{H}}_{2} \mathrm{OH}\right) .{ }^{13} \mathrm{C}-\mathrm{NMR}\left(101 \mathrm{MHz}, \mathrm{CDCl}_{3}\right) \delta 132.21$ (d, J $\left.2.7 \mathrm{~Hz}, \mathrm{C}_{4}, \mathrm{C}_{4^{\prime}}\right), 131.47$ (d, J $9.3 \mathrm{~Hz}$, $\left.\mathrm{C}_{2}, \mathrm{C}_{6}\right), 130.90\left(\mathrm{~d}, J 96.7 \mathrm{~Hz}, \mathrm{C}_{1}\right), 128.69$ (d, J $\left.11.6 \mathrm{~Hz}, \mathrm{C}_{3}, \mathrm{C}_{5}\right), 61.25$ (d, J $82.8 \mathrm{~Hz}, \mathrm{C}_{7}$ ).

(Diphenylphosphoryl)methyl 4-methylbenzenesulfonate (12). In a dry and nitrogen-flushed two-necked flask fitted with a condenser was charged diphenylhydroxymethylphosphine oxide (4.45 g, $18.07 \mathrm{mmol}, 1 \mathrm{eq})$.

Dichloromethane was added $(50 \mathrm{~mL})$ and the solution was cooled to $0{ }^{\circ} \mathrm{C}$. Trimethylamine $(2.64 \mathrm{ml}, 18.97$ $\mathrm{mmol}, 1.05 \mathrm{eq}$ ) was then added and the mixture was stirred for $30 \mathrm{~min}$ at room temperature. The solution was again cooled to $0^{\circ} \mathrm{C}$ and

p-toluenesulfonyl chloride $(3.61 \mathrm{~g}, 18.97 \mathrm{mmol}, 1.05 \mathrm{eq})$ was added. The reaction mixture was stirred for 30 min at $0^{\circ} \mathrm{C}$ followed by overnight at room temperature. Water $(100 \mathrm{~mL})$ was then added and the mixture was extracted with dichloromethane $(3 \times 200 \mathrm{~mL})$. After drying over magnesium sulfate and concentration, a white solid was obtained (6.95 g, yield 100\%). ${ }^{31} \mathrm{P}-\mathrm{NMR}\left(162 \mathrm{MHz}, \mathrm{CDCl}_{3}\right) \delta 25.81$ (s). ${ }^{1} \mathrm{H}-\mathrm{NMR}(400 \mathrm{MHz} \mathrm{CDCl})_{3} \delta$

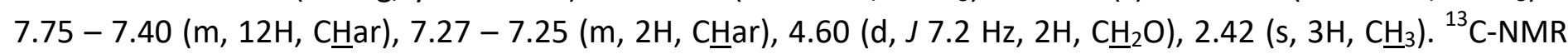
$\left(101 \mathrm{MHz}, \mathrm{CDCl}_{3}\right) \delta 145.67\left(\mathrm{~s}, \mathrm{C}_{8}\right), 132.95$ (d, J $\left.12.8 \mathrm{~Hz}\right), 131.60$ (d, J $\left.9.8 \mathrm{~Hz}\right), 130.62$ (d, J 9.8 Hz), $130.11(\mathrm{~s})$, 129.65 (s), 128.99 (s), 64.85 (d, J $82.1 \mathrm{~Hz}), 21.91$ (s, $\left.\mathrm{C}_{14}\right)$.

(Bromomethyl)diphenylphosphine oxide (13). In a 500-mL two-necked flask fitted with a condenser were charged acetonitrile $(240 \mathrm{~mL})$, the diphenylphosphorylmethyl-p-toluenesulfonate $(5.33 \mathrm{~g}, 12.93 \mathrm{mmol}, 1 \mathrm{eq})$ and sodium bromide $(5.67 \mathrm{~g}, 55.17 \mathrm{mmol}, 4 \mathrm{eq})$. The mixture was heated at $100^{\circ} \mathrm{C}$ for one week. After filtration and evaporation of the solvent, the solid obtained was recrystallized in ethanol ( 3 times). A white solid was obtained (2.65 g, yield 65\%).

${ }^{31}$ P-NMR (162 MHz, CDCl $) \delta 27.15$ (s). ${ }^{1} \mathrm{H}-\mathrm{NMR}\left(400 \mathrm{MHz}, \mathrm{CDCl}_{3}\right) \delta 7.83-7.75$ (m, 4H, Char), $7.62-7.45$ (m, $6 \mathrm{H}, \mathrm{C}$ ㅂar), 3.80 (d, J $5.7 \mathrm{~Hz}, 2 \mathrm{H}, \mathrm{CH}_{2} \mathrm{Br}$ ). ${ }^{13} \mathrm{C}-\mathrm{NMR}\left(101 \mathrm{MHz}, \mathrm{CDCl}_{3}\right) \delta 132.70\left(\mathrm{~d}, J 2.8 \mathrm{~Hz}, \mathrm{C}_{4}\right), 131.57$ (d, J $9.4 \mathrm{~Hz}$, $\left.C_{2}, C_{6}\right), 130.10$ (d, J $\left.104.9 \mathrm{~Hz}, C_{1}, C_{1^{\prime}}\right), 128.89$ (d, J $12.2 \mathrm{~Hz}, C_{3}, C_{5}$ ), 23.65 (d, J $69.6 \mathrm{~Hz}, \mathrm{C}_{7}$ ). 
Diethyl ((diphenylphosphoryl)methyl)phosphonate (14). In a dry and nitrogen-flushed 25-mL three-necked flask equipped with a Dean Stark apparatus and condenser were charged bromométhyldiphenylphosphine oxide (1.04 g, $3.53 \mathrm{mmol}, 1 \mathrm{eq})$ and triethylphosphite $(0.72 \mathrm{~mL}, 4.24 \mathrm{mmol}, 1.2 \mathrm{eq})$. The mixture was heated at $150^{\circ} \mathrm{C}$ for $48 \mathrm{~h}$ under stirring. After cooling, vacuum distillation to remove excess triethylphosphite was done. After silica gel column chromatography (AcOEt/MeOH, 9:1), the desired product was obtained as a white solid (0.86 g, yield 69\%). ${ }^{31} \mathrm{P}-\mathrm{NMR}(162 \mathrm{MHz}, \mathrm{DMSO}) \delta 22.60$ (d, J $\left.14.4 \mathrm{~Hz}\right), 20.48$ (d, J $\left.14.4 \mathrm{~Hz}\right) .{ }^{1} \mathrm{H}-\mathrm{NMR}(400 \mathrm{MHz}$,

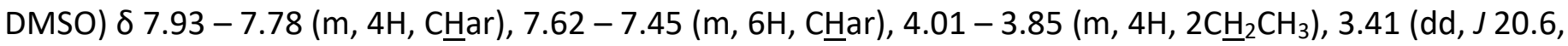
$13.9 \mathrm{~Hz}, 2 \mathrm{H}, \mathrm{PC}_{2} \mathrm{P}$ ), 1.07 (t, J 7.1 Hz, 6H, 2 $\left.\mathrm{CH}_{2} \mathrm{CH}_{3}\right) .{ }^{13} \mathrm{C}-\mathrm{NMR}(101 \mathrm{MHz}, \mathrm{DMSO}) \delta 134.53$ (dd, J $102.97 \mathrm{~Hz}, \mathrm{C}_{1}$ ), 131.47 (d, J $\left.2.7 \mathrm{~Hz}, \mathrm{C}_{4}\right), 131.10\left(\mathrm{~d}, J 9.8 \mathrm{~Hz}, \mathrm{C}_{2}, \mathrm{C}_{6}\right.$ ), 128.93 (d, J $12.0 \mathrm{~Hz}, \mathrm{C}_{3}, \mathrm{C}_{5}$ ), 61.40 (d, J $\left.6.0 \mathrm{~Hz}, \mathrm{C}_{8}, \mathrm{C}_{16}\right), 27.53$ (dd, J 129.8, $\left.62.3 \mathrm{~Hz}, \mathrm{C}_{7}\right), 15.95$ (d, J $\left.6.6 \mathrm{~Hz}, \mathrm{C}_{9}\right)$.

Diethyl (2-(4-(bromomethyl)phenyl)-1-(diphenylphosphoryl)ethyl)phosphonate (15a). ${ }^{31} \mathrm{P}-\mathrm{NMR}(162 \mathrm{MHz}$, $\mathrm{CDCl}_{3}$ ) $\delta$ 29.56-29.54 (bs), 23.00 (d, J $3.4 \mathrm{~Hz}$ ). ${ }^{1} \mathrm{H}-\mathrm{NMR}\left(400 \mathrm{MHz}, \mathrm{CDCl}_{3}\right) \delta 7.92-7.85$ (m, 2H), $7.80-7.70$ (m, $2 \mathrm{H}), 7.60-7.35(\mathrm{~m}, 6 \mathrm{H}), 7.15(\mathrm{~d}, J 8.2 \mathrm{~Hz}, 2 \mathrm{H}), 7.00(\mathrm{~d}, J 8.2 \mathrm{~Hz}, 2 \mathrm{H}), 4.41(\mathrm{~s}, 3 \mathrm{H}), 4.04-3.71(\mathrm{~m}, 4 \mathrm{H}), 3.41-$ $3.09(\mathrm{~m}, 2 \mathrm{H}), 2.08-1.98(\mathrm{~m}, 1 \mathrm{H}), 1.05(\mathrm{dt}, J 21.7,7.1 \mathrm{~Hz}, 6 \mathrm{H}) .{ }^{13} \mathrm{C}-\mathrm{NMR}\left(101 \mathrm{MHz}, \mathrm{CDCl}_{3}\right) \delta 139.85-139.53(\mathrm{~m}$, $\left.C_{1}\right), 136.39\left(s, C_{14}\right), 135.87\left(s, C_{11}\right), 131.23$ (dd, J 22.5, 9.4 Hz, C $), 129.06$ (d, J 30.1 Hz, C $), 128.76$ (d, J 6.4 Hz, $\mathrm{C}_{13}$ ), 128.61 (d, J $12.0 \mathrm{~Hz}, \mathrm{C}_{12}$ ), 128.29 (d, J $\left.12.3 \mathrm{~Hz}, \mathrm{C}_{4}\right), 62.46$ (dd, J 12.8, $6.8 \mathrm{~Hz}, \mathrm{C}_{8}$ ), 41.10 (dd, J 132.1, 58.3 Hz, $\left.C_{7}\right), 33.37\left(s, C_{16}\right), 30.03\left(s, C_{10}\right), 15.97\left(d, J 6.4 \mathrm{~Hz}, C_{9}\right)$.

Diethyl (1-(diphenylphosphoryl)-2-(p-tolyl)ethyl)phosphonate (15b). ${ }^{31} \mathrm{P}-\mathrm{NMR}\left(162 \mathrm{MHz}, \mathrm{CDCl}_{3}\right) \delta 29.86$ (d, J $4.1 \mathrm{~Hz}), 23.29$ (d, J 4.1 Hz). ${ }^{1} \mathrm{H}-\mathrm{NMR}\left(400 \mathrm{MHz}, \mathrm{CDCl}_{3}\right) \delta 8.00-7.85(\mathrm{~m}, 2 \mathrm{H}), 7.85-7.65(\mathrm{~m}, 2 \mathrm{H}), 7.54-7.33(\mathrm{~m}$, $6 \mathrm{H}), 6.92(\mathrm{q}, \mathrm{J} 8.1 \mathrm{~Hz}, 4 \mathrm{H}), 4.04-3.73(\mathrm{~m}, 4 \mathrm{H}), 3.35-2.95(\mathrm{~m}, 2 \mathrm{H}), 2.24(\mathrm{~s}, 3 \mathrm{H}), 1.27(\mathrm{~m}, 1 \mathrm{H}), 1.04(\mathrm{dt}, \mathrm{J}$ 19.0, $7.1 \mathrm{~Hz}, 6 \mathrm{H}) .{ }^{13} \mathrm{C}-\mathrm{NMR}\left(101 \mathrm{MHz}, \mathrm{CDCl}_{3}\right) \delta 136.48$ (dd, J 8.1, $4.4 \mathrm{~Hz}, \mathrm{C}_{1}$ ), $135.80\left(\mathrm{~s}, \mathrm{C}_{11}, \mathrm{C}_{14}\right), 131.60$ (dd, J 4.1, 2.9 $\mathrm{Hz}, \mathrm{C}_{2}$ ), 131.37 (d, J $\left.9.3 \mathrm{~Hz}, \mathrm{C}_{3}\right), 131.21$ (d, J $9.1 \mathrm{~Hz}, \mathrm{C}_{12}$ ), 128.71 (d, J $22.4 \mathrm{~Hz}, \mathrm{C}_{4}$ ), 128.32 (dd, J 28.7, $12.1 \mathrm{~Hz}$, $\mathrm{C}_{13}$ ), 62.23 (dd, J 11.7, $6.8 \mathrm{~Hz}, \mathrm{C}_{8}$ ), 41.52 (dd, J 131.6, $58.2 \mathrm{~Hz}, \mathrm{C}_{7}$ ), 29.92 (dd, J 4.1, 2.0 Hz, C 10 ), 20.95 (s, C 16 ), $15.99\left(\mathrm{dd}, \mathrm{J} 6.4,0.8 \mathrm{~Hz}, \mathrm{C}_{9}\right)$. HRMS (TOF MS ES ${ }^{+}$(m/z) : $[\mathrm{M}+\mathrm{H}]^{+}$calcd for $\mathrm{C}_{25} \mathrm{H}_{31} \mathrm{O}_{4} \mathrm{P}_{2}$ 457.1692. Found:

457.1711.

\section{Acknowledgements}

This work was partially supported by a grant of the French Embassy in Niamey (NIGER).

\section{Supplementary Material}

Copies of spectra of compounds are provided in the supplementary material file available on the Publisher's website.

\section{References}

1. Menschutkin, N. Ann. Chem. Pharmacol. 1865, 133, 317.

2. Cremers, S., Drake, M.T., Hal Ebetino, F., Bilezikian, J .P., Graham, R., Russell, G. Br J Clin Pharmacol. 2019, 85, 1052. https://doi.org/10.1111/bcp.13867 
3. Rogers, M. J .; Crockett, J . C.; Coxon, F. P.; Mönkkönen, J. Bone 2011, 49, 34. https://doi.org/10.1016/j.bone.2010.11.008

4. Papapoulos, S. E. Best Pract. Res. Clin. Endocrinol. Metab. 2008, 22, 831. https://doi.org/10.1016/j.beem.2008.07.001

5. Cremers, S.; Papapoulos, S. Bone 2011, 49, 42. https://doi.org/10.1016/j.bone.2011.01.014

6. Russell, R. G. G. Bone 2011, 49, 2. https://doi.org/10.1016/j.bone.2011.04.022

7. Russell, R. G. G.; Xia, Z.; Dunford, J . E.; Oppermann, U.; Kwaasi, A.; Hulley, P. A.; Kavanagh, K. L.; Triffitt, J. T.; Lundy, M. W.; Phipps, R. J .; Barnett, B. L.; Coxon, F. P.; Rogers, M. J .; Watts, N. B.; Ebetino, F. H. Ann. N. Y. Acad. Sci. 2007, 1117, 209.

https://doi.org/10.1196/annals.1402.089

8. Ebetino, F. H.; Hogan, A.-M. L.; Sun, S.; Tsoumpra, M. K.; Duan, X.; Triffitt, J . T.; Kwaasi, A. a; Dunford, J. E.; Barnett, B. L.; Oppermann, U.; Lundy, M. W.; Boyde, A.; Kashemirov, B. a; McKenna, C. E.; Russell, R. G. G. Bone 2011, 49, 20. https://doi.org/10.1016/i.bone.2011.03.774

9. Binnemans, K.; J ones, P. T.; Blanpain, B.; Van Gerven, T.; Yang, Y.; Walton, A.; Buchert, M. J . Clean. Prod. 2013, 51, 1 .

https://doi.org/10.1016/i.jclepro.2012.12.037

10. Massar, S; Ruberti, M. Resources Policy 2013, 38, 36. https://doi.org/10.1016/i.resourpol.2012.07.001

11. Sulzer-Mossé, S.; Tissot, M.; Alexakis, A. Org. Lett. 2007, 9, 3749. https://doi.org/10.1021/ol0514121

12. Viornery, C.; Péchy, P.; Boegli, M.; Aronsson, B.-O.; Descouts, P.; Grätzel, M. Phosphorus Sulfur Silicon Relat. Elem. 2002, 177 (1), 231. https://doi.org/10.1080/10426500108546631

13. Li, G.; Wu, M.; Liu, F.; J iang, J . Synthesis 2015, 47, 3783. https://doi.org/10.1055/s-0035-1560463

14. Barney, R. J .; Wasko, B. M.; Dudakovic, A.; Hohl, R. J .; Wiemer, D. F. Bioorg. Med. Chem. 2010, $18,7212$. https://doi.org/10.1016/i.bmc.2010.08.036

15. Göbel, R.; Richter, F.; Weichmann, Phosphorus Sulfur Silicon Relat. Elem. 1992, 73, 67.

16. SzaJ nman, S.H.; Rosso, V.S.; Malayil, L.; Smith, A.; Moreno, S.N.J .; Docampo, R.; Rodriguez, J . B. Org. Biomol. Chem. 2012, 10, 1424. https://doi.org/10.1039/C10B06602A

17. Greiner, I.; Grün, A.; Ludányi, K.; Keglevich, G. Heteroat. Chem. 2011, 22, 11. https://doi.org/10.1002/hc.20648

18. SzaJ nman, S.H.; Rosso, V.S.; Malayil, L.; Smith, A.; Moreno, S.N.J .; Docampo, R.; Rodriguez, J .B. Org. Biomol. Chem. 2012, 10, 1424. https://doi.org/10.1039/C10B06602A 